\title{
Short-course treatment in neurobrucellosis: A study in Iran
}

\author{
Persian Gulf Institute, Bushehr University of Medical Sciences, Bushehr, ${ }^{1}$ Namazi Hospital, University of Medical Sciences, Shiraz, \\ ${ }^{2}$ Bushehr University of Medical Sciences, Bushehr, Iran
}

\section{Address for correspondence:}

Dr. Farhad Abbasi,

Fateme-alzahra Hospital, Siraf St, Bushehr, Iran.

E-mail:f_abbasi55@yahoo.com

Received : 04-09-2010

Review completed : 26-09-2010

Accepted : 13-10-2010

\begin{abstract}
Neurobrucellosis is a rare neurological complication of brucellosis. This report describes 19 patients of neurobrucellosis and they accounted for $8 \%$ of all cases of brucellosis admitted to Shiraz University Hospitals over a period of eight years. Headache, fever, fatigue, drowsiness and neck stiffness were the common clinical features. Cerebrospinal fluid (CSF) showed pleocytosis in 100\%, elevated protein levels in $89 \%$ and low glucose level in $47 \%$ of the patients. All the patients improved with specific antibiotic treatment. Of the 19 patients, $10(52.5 \%)$ patients received treatment for 8 to 28 weeks. Duration of antibiotic treatment was: 8 - 14 weeks in 8 (42\%) patients; 24-28 weeks in 2 (10.5\%) patients; 6 months in 7 (37\%) patients; 12 months in 1 (5.3\%) patient; and 18 months in 1 (5.3\%) patient. Clinicians in endemic areas should consider the likelihood of neurobrucellosis in patients with unexplained neurological and psychiatric symptoms.
\end{abstract}

Key words: Neurobrucellosis, short course, treatment

\section{Introduction}

Central nervous system (CNS) involvement is a serious complication of brucellosis ${ }^{[1]}$ Neurobrucellosis occurs in $5-10 \%$ of cases of brucellosis. ${ }^{[2]}$ Neurological complications can be categorized into two major groups: 1) those related to the acute-febrile phase of the illness, toxic-febrile neurobrucellosis and 2) those related to primary affection of central and peripheral nervous system by the brucella infection. ${ }^{[2]}$ CNS involvement is commonly acute and results mainly in meningoencephalitis, while peripherial nervous system (PNS) involvement may be either acute or chronic. ${ }^{[3]}$ In this article, we report the clinical characteristics and treatment strategies of 19 patients with neurobrucellosis.

\begin{tabular}{|l|l|}
\hline \multicolumn{2}{|c|}{ Access this article online } \\
\hline Quick Response Code: & Website: \\
\hline & PMID: \\
\cline { 2 - 2 } & \\
\hline
\end{tabular}

\section{Material and Methods}

This is a retrospective study of patients with neurobrucellosis admitted to two tertiary care hospitals in Shiraz, Iran over a period of eight years. Diagnostic criteria for neurobrucellosis were: 1) clinical features compatible with a known neurobrucellosis syndrome; 2) typical cerebrospinal fluid (CSF) (elevated protein concentration or pleocytosis) findings; 3) positive blood or CSF serology for brucellosis (e.g., agglutination test titers of $>1: 160$ in blood or any positive titer in CSF); 4) clinical improvement with a course of antibiotic therapy; and 5) clinical syndrome otherwise not explainable by an alternative diagnosis. Case records were reviewed and the data collected included clinical features, laboratory data, neuroimaging findings, treatment details, and outcome. Serum anti-brucella immunoglobulin G (IgG) and immunoglobulin $\mathrm{M}(\mathrm{IgM})$ antibody were checked for patients who had negative Coomb's Wright agglutination tests results by enzyme-linked immunosorbant assay (ELISA) method. Antibiotic treatment included: doxycycline, rifampin, trimethoprim-sulfamethoxazole, streptomycin, gentamicin and ceftriaxone. Treatment was discontinued after clinical assessment by the 
physicians when they were certain that the infection was controlled. All patients were followed for two years to find any signs and symptoms of relapse.

\section{Results}

Of the 235 patients with brucellosis admitted during the study period, $19(8 \%)$ patients had neurobrucellosis. Mean age was 38.1 years (range $18-70$ years) and there were $14(74 \%)$ were males. The mean duration of symptoms before admission was 8 weeks (range 1 week - 4 months). The most common clinical features were: headache (79\%), fever ( $84 \%)$, neck rigidity ( $42 \%)$, fatigue $(37 \%)$, altered mental status $(31 \%)$, speech disturbances $(21 \%)$, nausea and vomiting (15\%). Other less common clinical features were hearing loss, paraplegia, cerebellar ataxia, diplopia, photophobia, blurred vision, abnormal behavior, hypoesthesia, low back pain and right-side weakness. Ten $(58 \%)$ patients had focal neurological deficits.

CSF analysis showed elevated protein $(>45 \mathrm{mg} / \mathrm{dl})$ in $17(89 \%)$ patients. low glucose $(<40 \mathrm{mg} / \mathrm{dl}$ and/or CSF/ serum glucose ratio of $<0.4)$ in nine $(47 \%)$ patients. CSF WBC count was between 6 cells/dl and 3600 cells/dl with mean count 403 cells / dl. Fifteen (79\%) patients had lymphocyte predominance and four $(21 \%)$ patients had polymorphonuclear (PMN) predominance. CSF cultures and Gram-stains were negative in all patients. Of the six sera tested for anti-brucella antibody, $3(50 \%)$ were positive. Anti-brucella antibodies in the CSF by either ELISA or Coomb's Wright agglutination tests were tested in 9 patients that was positive in $6(67 \%)$ patients.

Three patients had electrodiagnostic evidence of peripheral neuropathy. Brain computed tomography (CT) scan was done in 12 patients; only one scan showed infarct in the anterior limb of left internal capsule. This patient had right hemiparesis. Brain magnetic resonance imaging (MRI) was performed in eight patients with normal brain CT scan. Scans in two patients showed abnormalities; (1) a-23-year old male with one history of hearing loss and three month history of severe headache showed mild hydrocephalus with right temporal atrophy; and (2) a-27-year old male with one week history of fever, headache, inappropriate speech, abnormal behavior, and seizures showed putamenal infarctions.

Eight (42\%) patients who presented with fever, headache and/or drowsiness and no focal deficits, received 8-14 weeks' antibiotic therapy (doxycycline, rifampin and trimethoprim-sulfamethoxazole or streptomycin or ceftriaxone). Two patients with hearing loss had received 24 and 28 weeks of antibiotics respectively (doxycycline, rifampin, trimethoprim-sulfamethoxazole and ceftriaxone for the first month). Both the patients improved significantly in their hearing as shown in the followup audiometric studies. One patient with lower extremity weakness and peripheral neuropathy received 1.5 year antibiotic therapy (doxycycline, rifampin and trimethoprim-sulfamethoxazole). One patient with headache and photophobia with normal brain CT scan and brain MRI received one month doxycycline, rifampin and streptomycin. Treatment was continued by doxycycline and rifampin for 11 months. Seven patients (37\%) had received six months' antibiotic therapy with doxycycline, rifampin and trimethoprimsulfamethoxazole or ciprofloxacin or gentamicin (ceftriaxone for the first month). All the patients improved without any sequela and none had relapse during the two years' follow-up. Follow-up was done by visiting the patients and also by telephone. There were no side-effects related to long-term aminoglycoside therapy. Mild transient elevation of liver enzyme occurred in some patients with rifampin. One patient died in the second year because of a cardiac event at the age of 70 .

\section{Discussion}

Brucellosis is a multisystem disease with a broad spectrum of clinical manifestations. ${ }^{[4]} \mathrm{It}$ is a common zoonosis in many parts of the world and is not uncommon in Iran. Neurobrucellosis accounted for $8 \%$ of all cases of brucellosis and is comparable to the reported frequency $5-10 \%$ in the earlier studies. ${ }^{[5,6]}$ Headache, fever, sweating, weight loss, and back pain are the predominant symptoms in neurobrucellosis ${ }^{[1,7]}$ Meningitis and meningoencephalitis are the most common forms of neurobrucellosis. ${ }^{[8]}$ Other neurological complications including myelitis, radiculoneuritis, brain abscess, epidural abscess, meningovascular syndromes, subarachnoid hemorrhage and psychiatric manifestations. ${ }^{[9]}$ In our study meningitis and meningoencephalitis were the most common presentations. In this study the main reasons for delay in the diagnosis were less severe symptoms and lack of awareness of neurobrucellosis among the practicing physicians in this part of the world.

Serum agglutination test is often used for screening and complement fixation or Coombs' test are confirmatory tests. ELISA for brucella is more sensitive and specific than other serological tests and it may replace other serological tests. ELISA may detect antibodies against brucella in the serum and CSF. ${ }^{[10-12]}$ A patient with neurobrucellosis may have negative serological markers of brucellosis in the CSF and serum. ${ }^{[13]}$ Lymphocytic pleocytosis and elevated proteins in CSF were the common abnormalities in our series. The diagnosis of neurobrucellosis can be considered despite negative 
CSF culture and serology, based on clinical response and resolution of CSF abnormality with anti-brucella treatment. ${ }^{[13]}$

Neurobrucellosis is a treatable disease with a favorable outcome. Doxycycline, rifampicin, ceftriaxone, trimethoprim sulfamethoxazole, ciprofloxacin and streptomycin have been found effective in neurobrucellosis. ${ }^{[14-16]}$ Duration of treatment can depend on patient's condition. If rapid improvement occurs we may shorten the duration of antibiotic therapy to 12 weeks and continue their treatment by clinical assessment. In the study by Bodur et al, ${ }^{[14]}$ all patients received antibiotic therapy with ceftriaxone, rifampicin and doxycycline initially, and after one month they were continued with rifampicin and doxycycline up to four months. Doxycycline (by mouth) plus rifampin (by mouth) with ceftriaxone (intravenously) were the most common antibiotics in the study by Demiraslan et al. ${ }^{[17]}$ Most of our patients received antibiotic treatment for 24 weeks and an acceptable response was seen within 12 weeks in seven $(36 \%)$ patients. Short course of antibiotic therapy may be an option in patients with meningoencephalitis with no focal deficits or with minimal deficits. Patients with longer duration of the disease or with significant neurological deficits may require antibiotic treatment for a longer duration. Neurobrucellosis has an excellent prognosis when treated early and appropriately. The neurologic sequelae may be minimal. The important prognostic factors are duration of the disease, virulence of the microorganism and timing of antibiotic therapy. Early diagnosis and early institution of treatment is associated with fewer sequelae.

\section{References}

1. Gul HC, Erdem H, Bek S. Overview of neurobrucellosis: A pooled analysis of 187 cases. Int J Infect Dis 2009;13:e339-43.

2. Karsen H, Akdeniz H, Karahocagil MK, Irmak H, Sünnetçioğlu M. Toxic-febrile neurobrucellosis, clinical findings and outcome of treatment of four cases based on our experience. Scand J Infect Dis 2007;6:1-6.

3. Solera J, Martinez-Alfaro E, Espinosa A. Recognition and optimum treatment of brucellosis. Drugs 1997;53:245-56.

4. Tekin-Koruk S, Duygu F, Gursoy B, Karaagac L, Bayraktar M. A rare case of seronegative neurobrucellosis. Ann Saudi Med 2010;30:412-4.

5. Ozkavukcu E, Tuncay Z, Seluk F, Erden I. An unusual case of neurobrucellosis presenting with unilateral abducens nerve palsy: Clinical and MRI findings. Diagn Interv Radiol 2009;15:236-8.

6. Dias MS, Morganho A, Passão V, Aguiar T, Pedrosa R. Neurobrucellosis. Report of 8 cases. Acta Med Port 1995;8:671-5.

7. Ranjbar M, Rezaiee AA, Hashemi SH, Mehdipour S. Neurobrucellosis: Report of a rare disease in 20 Iranian patients referred to a tertiary hospital. East Mediterr Health J 2009;15:143-8.

8. Haji-Abdolbagi M, Rasooli-Nejad M, Jafari S, Hasibi M, Soudbakhsh A. Clinical and laboratory findings in neurobrucellosis: Review of 31 cases. Arch Iran Med 2008;11:21-5.

9. Yilmaz M, Ozaras R, Mert A, Ozturk R, Tabak F. Abducent nerve palsy during treatment of brucellosis. Clin Neurol Neurosurg 2003;105:218-20.

10. Al Dahouk S, Tomaso H, Nickler K, Neubauer H, Frangoulidis D. Laboratory-based diagnosis of brucellosis a review of the literature. Part II: Serological tests for brucellosis. Clin Lab 2003;49:577-89.

11. Jochum T, Kliesch U, Both R, Leonhardi J, Br KJ. Neurobrucellosis with thalamic infarction: A case report. Neurol Sci 2008;29:481-3.

12. Miguel PS, Fernández G, Vasallo FJ, Hortas M, Lorenzo JR, Rodríguez I, et al. Neurobrucellosis mimicking cerebral tumor: Case report and literature review. Clin Neurol Neurosurg 2006;108:404-6.

13. Samdani PG, Patil S. Neurobrucellosis. Indian Pediatr 2003;40:565-8.

14. Bodur H, Erbay A, Akinci E, Colpan A, Cevik MA, Balaban N. Neurobrucellosis in an endemic area of brucellosis. Scand J Infect Dis 2003;35:94-7.

15. Kochar DK, Agarwal N, Jain N, Sharma BV, Rastogi A, Meena CB. Clinical profile of neurobrucellosis a report on 12 cases from Bikaner (north-west India). J Assoc Physicians India 2000;48:376-80.

16. Akdeniz H, Irmak H, Anlar O, Demirz AP. Central nervous system brucellosis: Presentation, diagnosis and treatment. J Infect 1998;36:297-301.

17. Demiraslan H, Metan G, Mese EA, Yildiz O, Aygen B, Sumerkan B, et al. Neurobrucellosis: An evaluation of a rare presentation of brucellosis from a tertiary care centre in Central Anatolia, Turkey. Trop Doct $2009 ; 39: 233-5$

Source of Support: Conflict of Interest: 
Copyright of Neurology India is the property of Medknow Publications \& Media Pvt. Ltd. and its content may not be copied or emailed to multiple sites or posted to a listserv without the copyright holder's express written permission. However, users may print, download, or email articles for individual use. 